\title{
Real Time PCR for Dientamoeba fragilis: a comparison between molecular and microscopical approach
}

\author{
Ettore De Canale', Maria Angela Biasolo',2, Andrea Tessari', Sabrina Bettanello', \\ Valeria Besutti',Carlo Mengoli',2 \\ 'Service of Microbiology and Virology, Azienda Ospedaliera di Padova; \\ ${ }^{2}$ Department of Histology, Microbiology and Medical Biotechnology, Università degli Studi di Padova. Padova, Italy
}

Key words: Dientamoeba fragilis, parasitology, fixed stool, direct wet mount, real-time PCR

Real time PCR per Dientamoeba fragilis: confronto tra l'approccio molecolare e l'esame microscopico

\section{SUMMARY}

Introduction. Dientamoeba fragilis is an intestinal protozoan parasite that is attracting growing interest. Several authors have associated the presence of this faecal agent to numerous intestinal and systemic clinical symptoms, even though asymptomatic carriers have been reported.Actually, to perform the coproparasitology exam for the identification of $D$. fragilis it is necessary to recur to an expert microscopist. Recently, several real-time TaqMan assays for the detection of $D$. fragilis has been reported. In this work we validate an original real-time PCR (TaqMan), re-evaluating the traditional microscopic diagnosis. Moreover, the potential for quantitative information, intrinsic of this molecular technique, was preliminarly explored.

Methods. Forty-six carriers of $D$. fragilis were selected from patients referred to the Service of Microbiology of Padova University Hospital. The parasite was preliminarily detected on several occasions in each patient using traditional microscopy. A control group included forty-two healthy volunteers. Real-time PCR was applied to a stool sample from each subject. On the same sample, gel-electrophoresis amplicon detection PCR and microscopic examination were also performed.

Results. The sensitivity of real-time PCR was 100\%, whereas microscopy showed $93 \%$ sensitivity (direct wet mount on fixed stools and trichrome stain), gel-electrophoresis PCR $76 \%$ and Giemsa stain $52 \%$. The specificity of all methods was $100 \%$. On the quantitative side, the frequency distribution of the protozoan load of the stools revealed a near to log-normal pattern is apparent for the data yielded by real-time PCR.

Conclusions. The real-time PCR was clearly superior to either the gel electrophoresis PCR or the traditional microscopic examination. Just one unpreserved stool sample was adequate to find the parasite.

The entire procedure could be performed within a few hours avoiding toxic dyes.

Received May I2, 2009

Accepted June 4, 2009

\section{INTRODUCTION}

Dientamoeba fragilis is an intestinal protozoon that is attracting growing interest. Several authors have associated the presence of this faecal agent to numerous intestinal and systemic clinical symptoms, even though asymptomatic carriers have been reported $(3,5,9,10,15,19,22)$. D. fragilis's infection may persist for years with a range of 1-630 weeks and it may evoke various symptoms, including diarrhoea and abdominal pain $(14,17)$. Diarrhoea is the most common find- ing in patients with acute illness, whereas abdominal pain was more common in children with chronic symptoms (2). D. fragilis has been reported from most areas of the world where careful surveys have been taken. Overall estimates for various countries can vary from $1.5 \%$ to $53 \%$, meanwhile the parasite prevalence among unselected population in developed countries range from $1.5 \%$ to $4.2 \%(25,29)$.

The interest for an accurate, reliable, and costeffective method to detect the parasite has to be

\section{Corresponding author: Ettore De Canale}

Servizio di Microbiologia e Virologia dell'Azienda Ospedaliera di Padova,

Via Giustiniani 2, 35I00 Padova, ITALY - Tel: 003904982 I305I; Fax: 003904982 I3054;

E-mail: ettore.decanale@sanita.padova.it 
compounded with the impact of $D$. fragilis in human pathology, which still deserves further investigation.

In our experience, Dientamoeba fragilis showed a mean prevalence of $2.3 \%$ among people referred to our centre between 2005-2007, most of all to referred non hospitalized patients. One hundred patient resulted positive with traditional microscopy reported abdominal pain (36\%), diarrhoea (10\%), stipsis (8\%), weight loss (8\%) and nausea $(6 \%)$. Skin rush and spread itch were reported respectively on $26 \%$ and $20 \%$ of interviewed patients, sometimes with no other gastrointestinal symptoms associated (unpublished observations). High prevalence of cutaneous symptoms has not been reported in literature yet, so further investigations are request to clarify the link between $D$. fragilis and this pain.

Actually, to perform the coproparasitology exam for the identification of $D$. fragilis it is necessary to consult an expert microscopist. The laboratory detection rate of the organism is greatly enhanced by use of preservative to fix stool specimens immediately after passage, examining a minimum of three stool specimens $(11,27)$.

Currently microscopy is considered to be the gold standard for diagnosis of $D$. fragilis infection and it can be performed only with permanent stains on freshly passed or fixed stool samples. The use of permanently stained smears is a recommended and common practice in North America (7), but few laboratories in Italy use these techniques, which probably leads to an under-representation of the true prevalence of the parasite. Such techniques are time consuming and require experienced laboratory personnel to interpret the stained smears, at times with about 200-300 oil immersion fields examined microscopically (8). Culture techniques are by far the most sensitive method of detecting $D$. fragilis but these are too complex and time-consuming for routine laboratories $(18,27,28)$.

Molecular methods have also been proposed. PCR procedures with gel electrophoresis amplicon detection has been described (16, 20). Recently, a real-time TaqMan assay was also reported $(12,22)$. The aim of the present study was to investigate the effectiveness of the DNA amplification approach to the diagnosis of $\mathrm{D}$. fragilis infection. Compared to the traditional methods, it was reasonable to expect an increase in the diagnostic accuracy by using a PCR procedure. In this work we validated the real-time TaqMan assay by adopting an original set of primers and probe, and re-evaluated the traditional microscopic diagnosis. Moreover, a step was done toward the exploitation of the potential to furnish quantitative information residing in real-time PCR. Indeed, in several instances concerning the infectious diseases, the quantitative assay of the infecting agent is often predictive of the clinical context.

\section{MATERIALS AND METHODS}

Patients and samples A group of 46 carriers of Dientamoeba fragilis was enrolled, selected among the patients referred to the Service of Microbiology of Padova University Hospital for routine parasitological examination of stool samples during 2005-2007 (case group). Most of these subjects, with an age included between 4 and 72 years old, were symptomatic, reporting recurrent episodes of diarrhoea (or soft stools) or abdominal pain (or discomfort). Interestingly, a lot of them reported cutaneous symptoms, like skin rush and pruritus, often association with intestinal pain. $D$. fragilis was preliminarily detected on several occasions in each patient by reference parasitological examination based on optical microscopy (7). A second group (control group) was enrolled including 42 healthy volunteers (medical school students of the University of Padova and their relatives). All the 42 volunteers declared not to be affected by abdominal or systemic symptoms.

All subjects included in the study were asked to collect at home a faecal sample and to split it into two aliquots. One aliquot was put in a bottle containing SAF (sodium acetate-acetic acid-formalin) and the other one in an empty bottle. The unpreserved aliquot was preserved at $4{ }^{\circ} \mathrm{C}$ and transported to the laboratory at most 12 hours after evacuation. The unpreserved aliquots were analysed using two molecular techniques (gel electrophoresis PCR and real-time PCR) and the traditional optical Giemsa stain microscopy. The preserved aliquots were analysed using direct wet preparations of fixed faecal samples and Trichrome stain microscopy (7). The optical microscopic detection was performed independently by three expert parasitologist and the conventional and real-time PCR by different molecular biologists.

\section{DNA extraction}

DNA was isolated using a Packard Multi PROBE II instrument, with the QI Amp DNA Blood Bio Robot 9604b kit (Qiagen), following the manufacturer instructions. A stool sample of approximately $100 \mathrm{mg}$ was washed one time with PBS and the pellet processed for DNA extraction.

\section{Conventional PCR method}

A conventional gel-electrophoresis PCR procedure was adopted according to Peek (16) with some modifications. All PCR products were also directly sequenced on both strands by use of a 
DNA analyzer (ABI PRISM 3100; Applied Biosystems, Foster City, CA).

\section{TaqMan real-time PCR}

Real-time PCR primers and probe were selected using Primer Express Software (Applied Biosystems, Foster City, CA) on the sequence from Dientamoeba fragilis ssu rRNA gene (accession number U37461). The forward (5DMB) and reverse (3DMB) primer sequences were (5'GGCGAAAGCATCTATCAAGTGTATT-3') and (5'-CGGCATCGTTTAAGGTAGGAAC-3') respectively. The fluorogenic probe (5'-ACCCGGGTCTCTGATCCGGTTGG-3') contained a fluorescent reporter dye (6-carboxyfluorescein, FAM) at the 5'-end and a fluorescent quencher dye (6-carboxy-tetramethyl-rhodamine, TAMRA) at the 3 '-end.

Real-time PCR reaction was performed in a $25-\mu \mathrm{l}$ volume comprised of $5 \mu \mathrm{l}$ of DNA solution, 12,5 $\mu \mathrm{l}$ of TaqMan master mix (Applied Biosystems, Foster City, CA.), $0.6 \mu \mathrm{M}$ of each primer and probe $0,2 \mu \mathrm{M}$. TaqMan PCR reaction was carried out in an ABI PRISM 7900 HT sequence detection system and DNA was amplified according to the following program: 1 cycle of $95^{\circ} \mathrm{C}$ for 10 min, followed by 45 cycles each of $95^{\circ} \mathrm{C}$ for $15 \mathrm{~s}$ and $60^{\circ} \mathrm{C}$ for $1 \mathrm{~min}$. In order to asses the absence of PCR inhibitors, an Human genomic b-globin DNA amplification TaqMan assay was performed in parallel for each sample with the same amount of extracted DNA $(5 \mu \mathrm{l})$ as previously described (1). A 101 bp PCR fragment obtained by amplification of a $D$. fragilis positive sample with 5DMB and 3DMB primers was ligated into the pCR2.1 vector (TA-cloning Kit, Invitrogen, Carlsbad, CA) according to the manufacturer's instruction and

Table I. List of bacteria, protozoa and helminths and viruses used to test the analytical specificity of the real-time PCR

\begin{tabular}{ll}
\hline $\begin{array}{l}\text { Microorganisms } \\
\text { (miscellaneous) }\end{array}$ & Human parasites \\
\hline Salmonella typhimurium & Entamoeba histolytica/dispar \\
\hline Shigella flexneri & Entamoeba coli \\
\hline Campylobacter jejuni & Blastocystis hominis \\
\hline Yersinia enterocolitica & Giardia lamblia \\
\hline Aeromonas hydrophila & Trichomonas hominis \\
\hline Salmonella enteritidis & Endolimax nana \\
\hline Staphylococcus aureus & Chilomastix mesnili \\
\hline Bacillus cereus & lodamoeba buetschlii \\
\hline Vibrio parahaemolyticus & Cryptosporidium sp. \\
\hline Escherichia coli serotype 0I57 & Ascaris lumbricoides \\
\hline Helicobacter pylori & Taenia saginata \\
\hline Clostridium difficile & Enterobius vermicularis \\
\hline Candida albicans & Strongyloides stercoralis \\
\hline Adenovirus & \\
\hline Rotavirus & \\
\hline
\end{tabular}

sequenced on both strands. Homology searches were done with the Blast program with default settings (at http://www.ncbi.nlm.nih.gov/blast/). Ten-fold serial dilutions containing $10^{6}-10^{7}$ copies of the resulting plasmid, pTADMB were used in duplicate to generate standard curve for quantification of the protozoan load in clinical samples. To rule out false negative results due to the presence of amplification inhibitors or poor quality of DNA extraction, an exogenous DNA was amplified in parallel for each sample. The procedure appeared capable to detect the target copies in the range 10 to $10^{6}$.

Analytical specificity of the real-time PCR was evaluated on selected clinical specimens obtained from patients not included in the aforementioned case or control group, resulted positive for intestinal pathogens or other microrganisms usually recovered from the human gastrointestinal tract. Bacteria and yeast DNAs were extracted from colonies isolated by culture, viral and parasite agents were analysed from fresh stool specimens (Table 1). Three samples of vaginal secretions positive for Trichomonas vaginalis were also tested. The effect of storage conditions on real-time PCR performances was investigated. Six specimens split in 2 aliquots, the first one preserved in SAF and the other one maintained at $4^{\circ} \mathrm{C}$ without fixative, were analysed by real-time PCR for various time intervals from 7 to 60 days after collection.

\section{Statistical evaluation}

The proportion of the (true) positive results in the case group (sensitivity) and the proportion of the (true) negative results in the control group (specificity) was calculated for all diagnostic methods employed (real-time PCR, conventional PCR, direct examination of fixed specimen, Trichromic stained microscopy, and Giemsa stained microscopy). Since the real-time PCR is quantitative, a non parametric correlation with the microscopy (direct examination of fixed specimen) was examined by the Receiver-OperatingCharacteristic (ROC) analysis.

In addition, the quantitative data of genomic copy (positive data) were submitted to a frequency distribution analysis.

\section{RESULTS}

Positive and negative results data and accuracy indexes (sensitivity and specificity) are reported in Table 2. Real-time PCR (TaqMan) resulted positive in all members of the case group and negative in all control subjects, exhibiting a full sensitivity and specificity. On the other hand, a lower sensitivity performance was obtained by the conventional PCR. 
Table 2. Dientamoeba fragilis: Microscopy and PCR

\begin{tabular}{lccccl}
\hline & $\begin{array}{l}\text { Direct smear } \\
\text {-fixed stools }\end{array}$ & $\begin{array}{l}\text { Trichrome } \\
\text { stain }\end{array}$ & Giemsa stain & $\begin{array}{l}\text { Conventional } \\
\text { PCR }\end{array}$ & $\begin{array}{l}\text { real-time } \\
\text { PCR }\end{array}$ \\
\hline Sensitivity & $93 \%(43 / 46)$ & $93 \%(43 / 46)$ & $52 \%(24 / 46)$ & $76 \%(35 / 46)$ & $100 \%(46 / 46)$ \\
\hline Specificity & $100 \%(42 / 42)$ & $100 \%(42 / 42)$ & $100 \%(42 / 42)$ & $100 \%(42 / 42)$ & $100 \%(42 / 42)$ \\
\hline
\end{tabular}

Microscopic examination of wet preparation (direct wet mount) carried out on a single fixed stool specimen (SAF) was positive for 43 out 46 Dientamoeba fragilis carriers, and negative in all control subjects, appearing highly sensitive and fully specific. Trichromic stain microscopy performed equally well as direct examination on fixed specimen but this method can't be assumed as the gold standard when performed on samples preserved in SAF. Giemsa's stain performed on unpreserved stool processed within 12 hours after emission revealed a low sensibility and allowed Dientamoeba fragilis identification only in 24 out of 46 specimens resulted positive when collected using liquid fixatives. However, when Giemsa's stain is performed within 1 hour from the faeces, its sensibility is similar to that of microscopic searches performed on feces collected in liquid fixative (unpublished observations). A reassuring aspect of all method is the full specificity observed in all instances. The 11 discordant specimens resulted positive for $D$. fragilis using realtime PCR and not confirmed by conventional PCR were amplified another time using 5DMB and $3 \mathrm{DMB}$ primers and the amplification products were sequenced on both strands, confirming that they all belonged to the protozoa. A full analytical specificity was assessed by RealTime PCR analysis on DNA samples extracted from several intestinal pathogens, which resulted negative for all the specimens (Table 1). The effect of storage on the PCR was further evaluated with six microscopically $D$. fragilis-positive stool samples stored a $4{ }^{\circ} \mathrm{C}$ for various time intervals. In all six unpreserved samples $D$. fragilis's DNA could be detected up to 8 week. No specimens fixed in SAF after 7 days gave any amplification product with real-time PCR and no specimens stained with Giemsa resulted positives after 7 days.

The ROC curve is shown in Figure II. As binary, reference variable was used the direct microscopic examination, whereas as quantitative variable under scrutiny was examined the $D$. fragilis genomic copy number as detected by real-time PCR. The area under the curve was very high (99.7\%, 95\% C. I. 99-100\%), close to the ideal value $100 \%$. This indicates that the real-time PCR correctly predicts the outcome of microscopy. A threshold could be located in the range 1-79 DNA copies/reaction tube (each reaction tube contained 1/20 of each $100 \mathrm{mg}$ faecal sample). Therefore, even the positive finding of a minimal genomic copy level (close to one) yielded by real-time PCR would appear strongly predictive for a positive microscopic test.

As far the frequency distribution of the quantitative data was concerned, a positive skewness was apparent, as confirmed by the difference between median (620 genomic copies) and mean (37.181 genomic copies). The distribution reshapes toward normality if the protozoan load is logtransformed. This is shown in Figure III.

\section{DISCUSSION}

The high sensitivity of the microscopy can be credited to the skill of the highly trained parasitologist who performed analysis, as noted by others as well (22). It has been reported that from unselected patients, examining microscopically a minimum of three stool specimens for the search of $D$. fragilis, the sensitivity of examination should be at least 93.3\% (11). The negative result obtained by the microscopic analysis of two persistent $D$. fragilis carriers can be explained with the intermittent course of the fecal elimination of this protozoa, and by the low parasite charge. Day-to-day shedding, sometime, is highly variable for trophozoites, which imposes the need for multiple sampling to detect $D$. fragilis by optical microscopic examination. The quantitative search of $D$. fragilis by real-time PCR confirmed a low genomic copy content in the samples obtained by these two patients.

When present, characteristic morphologic markers should be recognized such as the appendages that we named “ golf-club” (Figure I), which usually are not seen in faecal samples not collected in fixative liquid ( SAF) after some hours of emission, (6). The Trichromic stain is time consuming (8) and uses toxic substances that have to be adequately disposed of. Examination of samples after Giemsa stain had poor sensitivity because unpreserved stool samples normally are delivered to the laboratory several hours after emission, enough time for the $D$. fragilis's trophozoites to be undergoing an "autolysing" process especially if the stool is formed. In soft stool samples however, it is possible to recover them even after a long time (6).

The sensibility of real-time PCR was proven superior to the traditional parasite identification with microscopical approach and a fully specifici- 
ty was demonstrated with analysis performed on stool samples from 42 "healthy volunteers" and on the main human intestinal pathogens. No aspecific amplifications were observed also with Trichomonas vaginalis which share a common evolutionary linkage with Dientamoeba fragilis. Primers and probe for TaqMan PCR were designed with high care, in order to find out the primers and probe which better amplify $D$. fragilis DNA and which also share the highest number of mismatch in the sequences of human parasites and intestinal pathogens.

Molecular biology techniques offer the potential of a highly sensitive and specific alternative to traditional diagnostic approaches such as microscopy or culture techniques $(13,16,20,21$, 22). Nucleic acids analysis furthermore helps to overcome the main limitation of the optical microscopy approach, the lysis of $D$. fragilis's trophozoites, a process which starts a few minutes after passage.

We are proposing this new molecular technique, rapid and economically advantageous for whoever has the possibility to carry-out molecular test in real-time PCR.

The new test, other than being more efficient than the traditional diagnostic approaches, can be considered a valid alternative to the permanent stain, which is, at the moment, the reference test for Dientamoeba fragilis identification.

Real-time PCR was able to detect $D$. fragilis's nucleic acid 60 days after emission of faecal sample even in the absence of trophozoites detectable with microscopy. $D$. fragilis DNA was also suc-



Figure I. Two trophozoites of Dientamoeba fragilis observed at 1.000x by direct wet mount of fixed unstained stool. One top left presents a typical round shaped.

The other below right shows a cytoplasmic extension towards the top that we named "golf club"

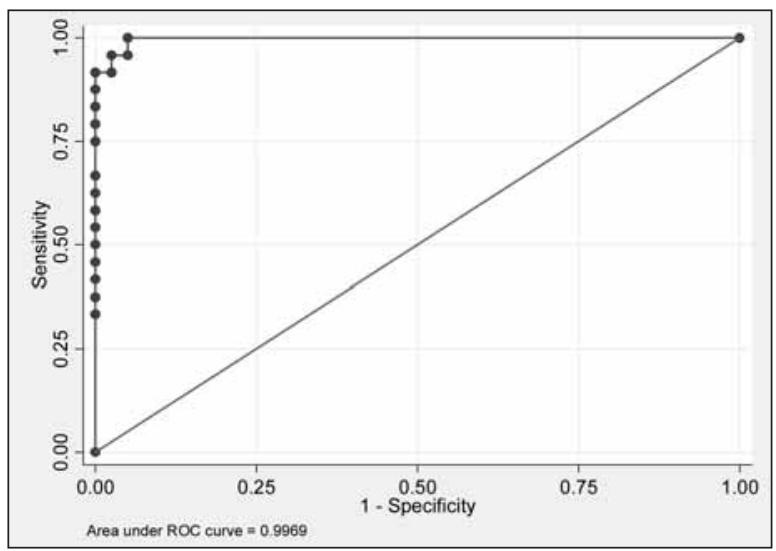

Figure II. ROC curve analysis. As binary, reference variable was used the direct microscopic examination, whereas as quantitative variable under scrutiny was examined the Dientamoeba fragilis genomic copy number as detected by real-time $P C R$.

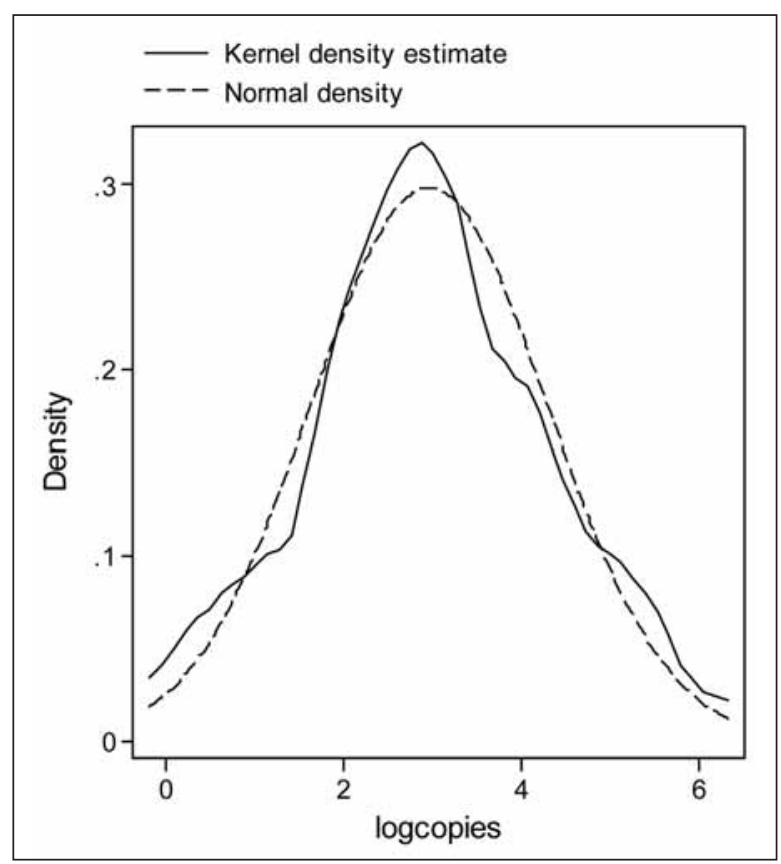

Figure III. Frequency distribution of the protozoan load of the stools, positive data. Data (Dientamoeba fragilis genomic copy counts) have been transformed into their decimal logarithms. For comparison, a normal distribution curve is shown as well. A near to log-normal pattern is apparent for the data yielded by real-time PCR.

cessfully amplified even from stools frozen at $70^{\circ} \mathrm{C}$ for more than a year. Those results suggest that fresh fecal sample cold be suitable for multiple target analysis performed in different days on the same sample and that it may also become a suitable instrument for genotyping and retrospective epidemiological analysis in parasitology, by the fact that a very large amount of DNA cold be easily recovered even after several months.

Real time PCR for $D$. fragilis can be performed within a few hours, it demonstrated an high sensitivity which may suggest that just one unpre- 
served stool sample could be sufficiently sensitive to find out the parasite and it can also be used to further investigate the epidemiology of $D$. fragilis and hence to enlighten long time darkness around this protozoa $(4,13,24)$.

\section{REFERENCES}

1. Biasolo MA, Calistri A, Cesaro S, et al. Case report: kinetics of Epstein-Barr virus load in a bone marrow transplant patient with no sign of lymphoproliferative disease. Journal of Medical Virology 2003; 69: 220-224

2. Chan FTH, Guan MX, Mackenzie AMR. Application of indirect Immunofluorescence to detection of Dientamoeba fragilis trophozoites in fecal specimens. J Clin Microbiol 1993; 31: 1710-1714

3. Crotti D, D’Annibale ML(2001). Dientamoeba fragilis and dientamoebiasis: Aspects of clinical parasitology and laboratory diagnosis. Parassitologia 2001; 43: $135-8$

4. Crotti D, Sensi M, Crotti S, et al. Dientamoeba fragilis in swine population: A preliminary investigation. Vet Parasitol 2007; 145: 349-351

5. Cuffari C, Oligny L, Seidman EG. Dientamoeba fragilis masquerading as allergic colitis. J Pediatr Gastroenterol Nutr 1998; 26: 16-20

6. De Canale E, Tessari A, Campion L, et al. Dientamoeba fragilis: is it really fragile? Approach to specimen handling and rapid microscopic diagnosis. Parassitologia 2003; 45: 19-22

7. Garcia LS. Laboratory methods for diagnosis of parasitic infection. In: Baron EJ, Peterson LR, Finegold SM, eds. Bailey and Scott's diagnostic microbiology. 9th edn. St Louis: Mosby, 1994: 776-861

8. Garcia LS, eds. Diagnostic Medical Parasitology. 4th edn. Washington DC: ASM Press, 2001

9. Girginkardesler N, Coskum S, Cuneyt Balcioglu I, et al. Dientamoeba fragilis, a neglected cause of diarrhea, successfully treated with secnidazole. Clin Microbiol Infect 2003; 9: 110-113

10. Grendon JH, DiGiacomo RF, Frost FJ. Desciptive features of Dientamoeba fragilis infections. Journal of Tropical Medicine and Hygene 1995; 98: 309-315

11. Hiatt RA, Markell EK, Ng E. How many stool examinations are necessary to detect pathogenic intestinal protozoa?. Am J Trop Med Hyg 1995; 53: 36-39

12. Verweij Jaco J, Mulder B, Poell B, et al. Real-time PCR for the detection of Dientamoeba fragilis in fecal samples. Mol and Cellular Probes 2007; (21) 400-404

13. Johnson EH, Windsor JJ, Clark CG. Emerging from Obscurity: Biological, Clinical, and Diagnostic Aspects of Dientamoeba fragilis. Clin Microbiol Rev 2004; 3: 553-570

14. Norberg A, Nord CE, Evengard B. Dientamoeba fragilis-a protozoal infection which may cause severe bowel distress. Clin Microbiol Infect 2003; 9: 65-68
15. Oxner R, Paltridge G, Champman B, et al. Dientamoeba fragilis: a bowel pathogen? New Zeland Medical Journal 1987; 100 :64-5

16. Peek R, Reedeker FR, Van Gool T. Direct Amplification and Genotyping of Dientamoeba fragilis from human stool specimens. J Clin Microbiol 2004; 42:631-635

17. Preiss U, Ockert G, Broemme S, et al. On the clinical importance of Dientamoeba fragilis infections in childhood. J Hyg Epidemiol Microbiol Immunol 1991; 35: 27-34

18. Sawangiaroen N, Luke R, Prociv P. Diagnosis by faecal culture of Dientamoeba fragilis infections in Australian patient with diarrhoea. Transactions of the Royal Society of Tropical Medicine and Hygiene 1993;87: 163-165

19. Spencer MJ, Garcia LS, Chapin MR. Dientamoeba fragilis an intestinal pathogen in children?. Am J Dis Child 1979; 133: 390-393

20. Stark D, Beebe N, Marriot D, et al. Detection of Dientamoeba fragilis in fresh stool specimens using PCR. International Journal for Parasitology 2005a; 35: 57-62

21. Stark D, Beebe N, Marriot D, et al. Prospective study of the prevalence, genotyping, and clinical relevance of Dientamoeba fragilis infections in an Australian population. J Clin Microbiol 2005b; 43: 2718-2723

22. Stark D, Beebe N, Marriot D, et al. Evaluation of three diagnostic methods, including real-time PCR, for detection of Dientamoeba fragilis in stool specimens. J Clin Microbiol 2006; 44: 232-5

23. Stark D, Beebe N, Marriot D, et al. Dientamoeba fragilis as cause of travelers' diarrhea: report of seven cases. J Travel Med. 2007; 14(1):72-3

24. Stark D, Phillips O, Peckett D, et al. Gorillas are a host for Dientamoeba fragilis: an update on the life cycle and host distribution. Vet Parasitol. 2008; 151(1):21-6

25. Van Gool T, Weijts R, Lommerse E, et al. Triple faeces test: an effective tool for detection of intestinal parasites in routine clinical practice. European Journal of Clinical Microbiology and Infectious Disease 2003; 22: 284-290

26. Windsor JJ, Rafay AM, Shenoy AK, et al. Incidence of Dientamoeba fragilis in faecal samples submitted for routine microbiological analysis. Br J Biomed Sci 1998; 55: 172-175.

27. Windsor JJ, Johnson EH. Dientamoeba fragilis: the unflagellated human flagellate. Br J Biomed Sci 1999; 56: 293-306

28. Windsor JJ, Macfarlane L, Hughes-Thapa G, et al. Detection of Dientamoeba fragilis by culture. $\mathrm{Br} J$ Biomed Sci 2003; 60 :79-83

29. Yang J, Scholten TH. Dientamoeba fragilis: A review with notes on its epidemiology, pathogenicity, mode of transmission, and diagnosis. Am J Trop Med Hyg 1977; 26: 16-22 